\title{
Coating Iron Oxide Nanoparticles with Chitosan for Targeted Delivery of Nanocurcumin
}

\author{
Zahra Hami ${ }^{1,}{ }^{*}$ \\ ${ }^{1}$ Toxin Research Center, AJA University of Medical Sciences, Tehran, Iran \\ "Corresponding author: Toxin Research Center, AJA University of Medical Sciences, Tehran, Iran. Email: hamiz1357@yahoo.com
}

Received 2020 April 12; Accepted 2020 April 14.

\begin{abstract}
Background: Curcumin is an active non-toxic component of turmeric and is a substance with strong anti-inflammatory and antioxidant properties. Iron, as a micronutrient, plays an important role in physiological processes. Chitosan is a natural polymer derived from chitin and is known as a biocompatible product due to its high biocompatibility, non-toxicity, and biodegradation to harmless products.

Objectives: This study aimed to design and synthesize a modified magnetic nanoparticle to be used as a targeted drug delivery system.

Methods: Nanocurcumin loading was performed on the surface of chitosan-modified iron magnetic nanoparticles in two stages. In the first stage, chitosan was loaded on the surface of iron magnetic nanoparticles as a coating polymer. In the next step, the final reaction took place by the loading of nanocurcumin on the surface of magnetic iron nanoparticles modified by chitosan.

Results: The results of transmission electron microscopy and scanning electron microscopy demonstrated the structure, morphology, physicochemical properties, and presence of nanocurcumin layers on chitosan in nanoparticles with a diameter of $20 \mathrm{~nm}$. In the Fourier transform infrared spectroscopy, the iron-oxygen peak indicated the magnetic nanoparticles of iron, and the oxygenhydrogen peak showed nanocurcumin layers on chitosan. Moreover, X-ray spectroscopy confirmed the presence of these elements in the final composition by showing iron, carbon, oxygen, and nitrogen peaks.

Conclusions: The results of this study showed that nanocurcumin was successfully loaded on the surface of magnetic iron nanoparticles modified by chitosan.
\end{abstract}

Keywords: Chitosan, Curcumin, Iron, Nanoparticles, Spectrophotometry

\section{Background}

The drug delivery system (DDS), based on nanotechnology has significantly improved drug therapy due to inducing pharmacokinetic changes, increasing the duration of action, reducing toxicity, and increasing the half-life of drugs. All of these benefits are possible to reach with targeted drug delivery by using magnetic nanoparticles as carriers.

Drug delivery systems are more efficient than other nanostructures due to their unique properties (1). The effect of nanoparticles' shape on their catalytic activity is also undeniable, and many studies have synthesized nanoparticles with specific shapes. Nanostructures such as multifaceted nanoparticles that have more available surfaces show much more catalytic activity than nanoparticles that have fewer facets $(2,3)$. These nanostructures can exhibit high catalytic properties and are widely used in chemical transformation as catalysts (4). Metal nanoparticles can be used in the pharmaceutical industry, and their magnetic properties are employed in the manufacturing of electronic devices and highly sensitive sensors (5-8). Due to the importance of metal nanoparticles and their applications in various fields of experimental sciences, many advances have occurred in recent years in the synthesis and stabilization of these nanoparticles on different platforms, which is essential to prevent adhesion and compaction of nanoparticles (9-11). Curcumin, a polyphenolic compound derived from turmeric, has different pharmacological effects such as anti-inflammatory, antioxidant, and anticoagulant activity. Phase I clinical trials have shown that curcumin at high doses (12 g per day) is safe for humans (12). Therefore, in this study, we developed a relatively simple, rapid, and less time-consuming method for the synthesis of curcumin-loaded chitosan magnetic nanoparticles. 


\section{Objectives}

This study aimed to design and synthesize a modified magnetic nanoparticle as a targeted drug delivery system.

\section{Methods}

We added $5.838 \mathrm{~g}$ ferric chloride hexahydrate $\left(\mathrm{FeCl}_{3} \cdot 6 \mathrm{H}_{2} \mathrm{O}\right)$ and $2.147 \mathrm{~g}$ ferric chloride tetrahydrate $\left(\mathrm{FeCl}_{2} \cdot 4 \mathrm{H}_{2} \mathrm{O}\right)$ to deionized water and stirred at $85^{\circ} \mathrm{C}$ by a mechanical stirrer. A black precipitate of magnetic nanoparticles was formed after adding ammonium hydroxide to the reaction mixture and then dispersed into $80 \mathrm{mg}$ of the prepared chitosan (CS) solution. The diluted compound was then sonicated for $30 \mathrm{~min}$ and neutralized with sodium hydrogen carbonate solution. Drops of the aqueous solution of iron-chitosan magnetic nanoparticles were added to nanocurcumin dissolved in acetonitrile. The suspension was then given $24 \mathrm{~h}$ to be stirred with a mechanical mixer at 12,000 rpm. This caused nanocurcumin to penetrate the polymer layer of shell-core nanoparticles. The obtained nanoparticles were washed in water to remove unloaded nanocurcumin. The prepared nanostructure was evaluated by Fourier transform infrared (FT-IR) spectroscopy, scanning electron microscopy (SEM), transmission electron microscopy (TEM), and X-ray diffraction (XRD) analysis. Loaded nanocurcumin was measured by a spectrophotometer (NanoDrop Technologies, Wilmington, DE, USA) at $450 \mathrm{~nm}$.

\section{Results}

Magnetic nanoparticles of iron oxide were synthesized using ferro/ferric chloride salts, and then their surfaces were coated with chitosan. Finally, nanocurcumin-loaded magnetic nanoparticles with the coat of chitosan were synthesized. The structure of prepared nanoparticles was confirmed by the FT-IR spectrum (Figure 1), SEM (Figure 2), and TEM (Figure 3) images. The FT-IR spectrum showed that nanocurcumin was successfully loaded on the surface of chitosan-modified iron magnetic nanoparticles. The stretching vibration absorption band at $3,421 \mathrm{~cm}^{-1}$ showed the hydroxy bond binding the nanocurcumin layer to chitosan. The absorption bands at $565 \mathrm{~cm}^{-1}$ and $635 \mathrm{~cm}^{-1}$ are related to the iron-oxygen bond in iron oxide. The peaks appearing in 3,400 $\mathrm{cm}^{-1}$ and $2,930 \mathrm{~cm}^{-1}$ regions are related to oxygen-hydrogen stretching vibrations, respectively, in nanocurcumin. The successful stabilization of carbonyl groups was observed with the appearance of an absorption band at $1,628 \mathrm{~cm}^{-1}$. The peak at $1,430 \mathrm{~cm}^{-1}$ is related to the carbon double bond. The carbon-oxygen bond in the chitosan compound also appeared in the $1,155 \mathrm{~cm}^{-1}$ absorption band. The SEM image of the synthesized iron-chitosan magnetic nanoparticles loaded with nanocurcumin confirms that nanocurcumin was made up of uniform particles in nanometer size. The particles were not completely spherical. In addition, particle condensation was observed in some areas, possibly due to the magnetic interaction between particles. Another reason for successful polymerization was the increase in the size of nanoparticles compared to iron oxide nanoparticles (Figure 2). The evaluation of the electron microscopy image of iron-chitosan magnetic nanoparticles loaded with nanocurcumin revealed that the nanoparticles formed a spherical shape on iron oxide nanoparticles containing organic-mineral shells. The size of synthesized nanoparticles is $20 \mathrm{~nm}$, as shown in electron microscopy images. The presence of the polymorphism layer of chitosan nanocurcumin is also shown in the figure.

The X-ray diffraction energy spectrum provided qualitative data on the distribution of various chemical elements in iron-chitosan magnetic nanoparticles loaded with nanocurcumin (Figure 4). Moreover, X-ray energy spectroscopy was used to determine the elements in curcumin-loaded nanoparticles on the surface of ironchitosan magnetic nanoparticles. Iron, carbon, nitrogen, and oxygen peaks confirmed the presence of these elements in the prepared nanoparticles.

\section{Discussion}

According to Allegra et al. study, curcumin is known as a potent anticancer, antioxidant, and anti-inflammatory agent (13). The study of anti-proliferative and anticancer effects of nanocurcumin by Khaniki et al. (14) showed the benefits of this natural and inexpensive compound for rat's colon cancer. According to some studies, nanocurcumin inhibits the expression and production of cyclooxygenase-2 (COX-2) (15). One of the most interesting properties of turmeric is its anti-inflammatory properties, so that turmeric is used as a topical and oral medicine to treat inflammation in Chinese medicine (16). The results of our study showed that the synthesized nanoparticles were spherical, and their sizes increased in the process of chitosan coating and curcumin loading, which is a reason for the successful polymerization. Based on the results obtained from the electron microscopy image of iron-chitosan magnetic nanoparticles loaded with nanocurcumin, the nanoparticles formed spherically 


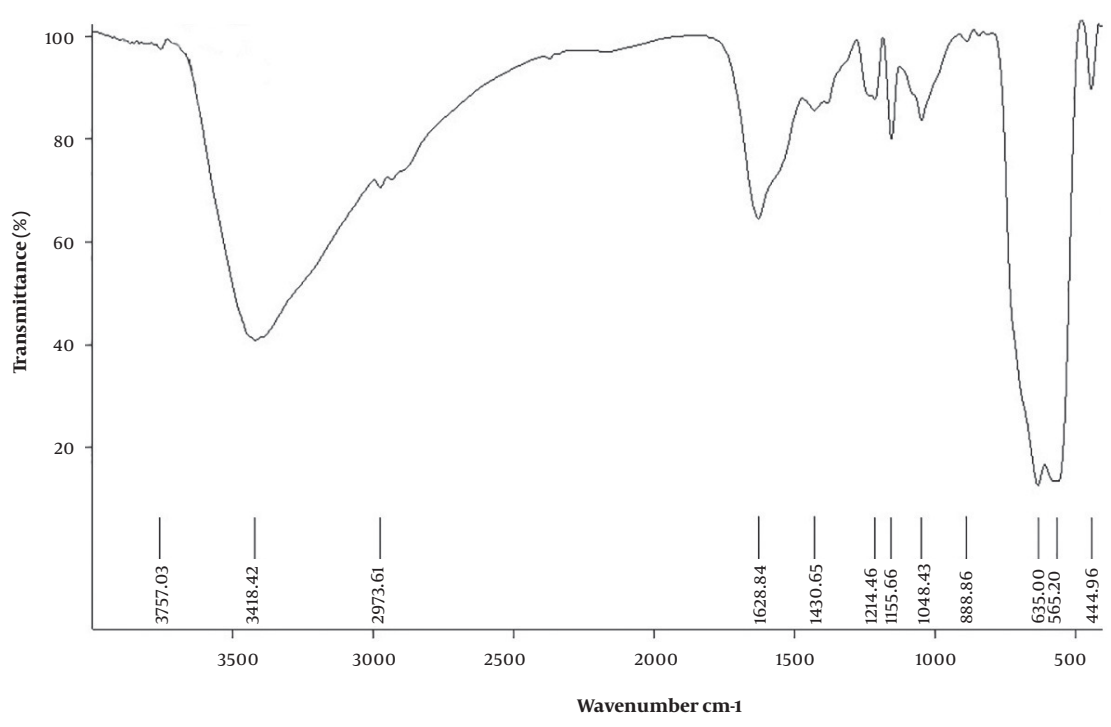

Figure 1. FTIR spectrum of curcumin-loaded CS- $\mathrm{Fe}_{3} \mathrm{O}_{4}$ nanoparticles
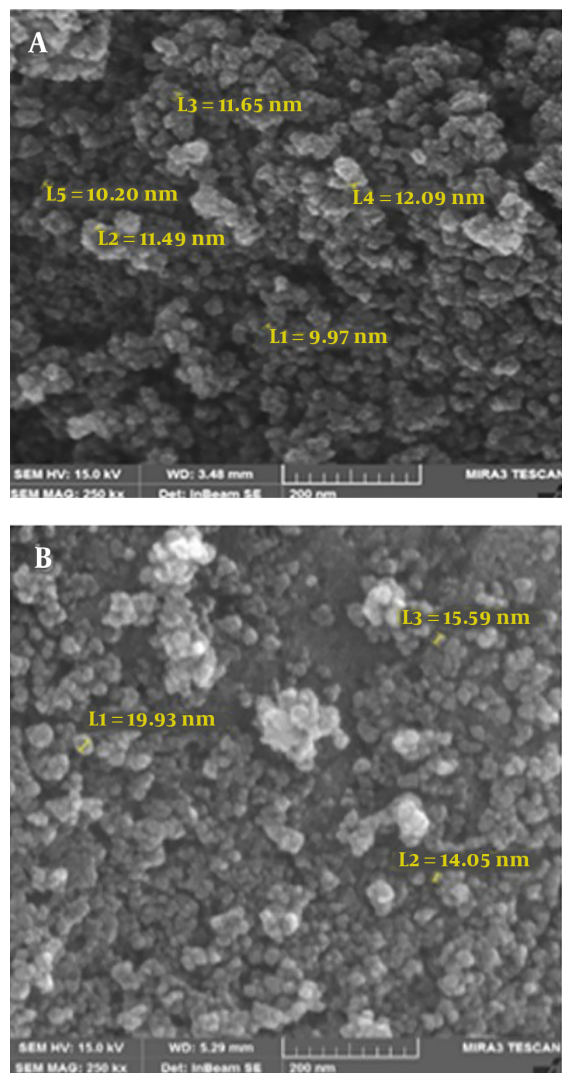

on iron-oxide nanoparticles containing organic-mineral shells with the size of $20 \mathrm{~nm}$. The magnetic properties of iron-chitosan magnetic nanoparticles loaded with nanocurcumin were determined by a magnetic device, a vibration sample meter, showing that the magnetic saturation of iron-oxide nanoparticles at room temperature was $63.1 \mathrm{emu} . \mathrm{g}^{-1}$ as measured by the magnetic curve (data not shown). A clear decrease in magnetic saturation (35.3 emu. $\mathrm{g}^{-1}$ ) of iron-chitosan magnetic nanoparticles loaded with nanocurcumin occurred due to the stabilization of polymeric groups on the nanoparticle surface. This evidence confirmed that nanocurcumin had been successfully loaded on the surface of chitosan-modified iron magnetic nanoparticles.

\subsection{Conclusions}

Nanocurcumin-loaded iron magnetic nanoparticles modified by chitosan were prepared, with active targeting capability. The prepared nanostructures modified by chitosan with appropriate size have a great potential for in vivo applications such as antioxidative, anticarcinogenic, and anti-inflammatory activities.

\section{Footnotes}

Authors' Contribution: Study concept and design, analysis and interpretation of data, drafting of the manuscript, Figure 2. SEM images of $\mathrm{A}, \mathrm{Fe}_{3} \mathrm{O}_{4}$; and B, curcumin-loaded $\mathrm{CS}-\mathrm{Fe}_{3} \mathrm{O}_{4}$ nanoparticles and critical revision of the manuscript for important intellectual content: Zahra Hami 

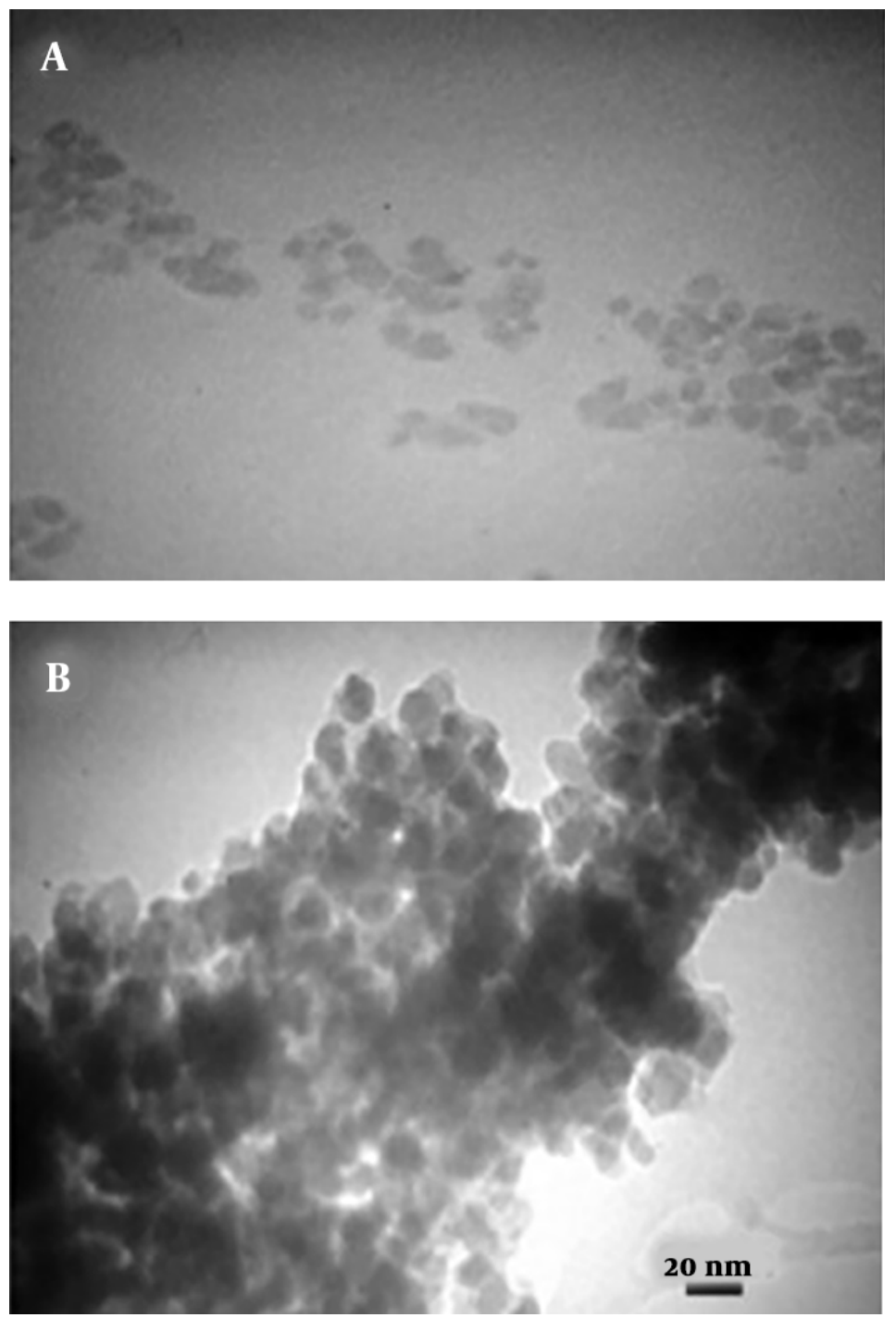

Figure 3. TEM images of $\mathrm{A}, \mathrm{Fe}_{3} \mathrm{O}_{4}$; and $\mathrm{B}$, curcumin-loaded $\mathrm{CS}-\mathrm{Fe}_{3} \mathrm{O}_{4}$ nanoparticles

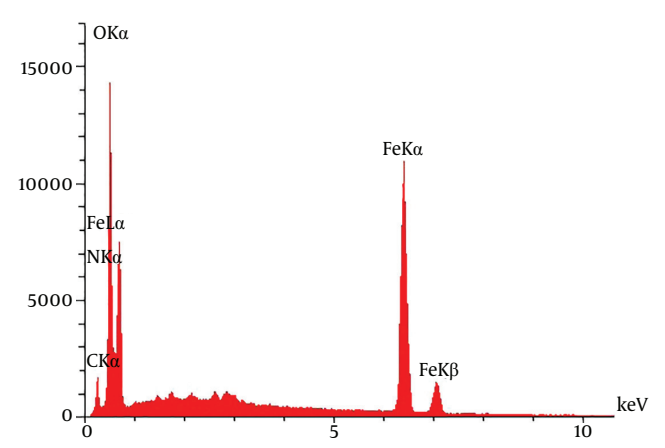

Figure 4. XRD patterns of curcumin-loaded $\mathrm{CS}-\mathrm{Fe}_{3} \mathrm{O}_{4}$ nanoparticles
Conflict of Interests: The authors declare that they have no conflict of interest.

Ethical Approval: The study procedures were approved by the Ethics Committee of AJA University of Medical Sciences, Tehran, Iran

Funding/Support: This study was supported by the AJA University of Medical Sciences, Tehran, Iran.

\section{References}

1. Kim JE, Shin JY, Cho MH. Magnetic nanoparticles: An update of application for drug delivery and possible toxic effects. Arch Tox- 
icol. 2012;86(5):685-700. doi: 10.1007/s00204-011-0773-3. [PubMed: 22076106].

2. Wang JJ, Zeng ZW, Xiao RZ, Xie T, Zhou GL, Zhan XR, et al. Recent advances of chitosan nanoparticles as drug carriers. Int J Nanomedicine. 2011;6:765-74. doi: 10.2147/IJN.S17296. [PubMed: 21589644]. [PubMed Central: PMC3090273].

3. Ciriminna R, Sciortino M, Alonzo G, Schrijver A, Pagliaro M. From molecules to systems: Sol-gel microencapsulation in silica-based materials. Chem Rev. 2011;111(2):765-89. doi: 10.1021/cr100161x. [PubMed 20726523].

4. Morgan RA, Yang JC, Kitano M, Dudley ME, Laurencot CM, Rosenberg SA. Case report of a serious adverse event following the administration of $\mathrm{T}$ cells transduced with a chimeric antigen receptor recognizing ERBB2. Mol Ther. 2010;18(4):843-51. doi: 10.1038/mt.2010.24. [PubMed: 20179677]. [PubMed Central: PMC2862534]

5. Nemamcha A, Rehspringer JL, Khatmi D. Synthesis of palladium nanoparticles by sonochemical reduction of palladium(II) nitrate in aqueous solution. I Phys Chem B. 2006;110(1):383-7. doi: 10.1021/jp0535801. [PubMed: 16471546].

6. Chen O, Zhao J, Chauhan VP, Cui J, Wong C, Harris DK, et al. Compact high-quality CdSe-CdS core-shell nanocrystals with narrow emission linewidths and suppressed blinking. Nat Mater. 2013;12(5):44551. doi: 10.1038/nmat3539. [PubMed: 23377294]. [PubMed Central PMC3677691].

7. Wu WT, Shi L, Zhu Q, Wang Y, Pang W, Xu G, et al. Novel dendritic nanostructures: Self-assemblies of nanoparticles of poly(vinyl alcohol) coated Ag and/or Cu2O. Nanotechnology. 2006;17(8):1948-53. doi: 10.1088/0957-4484/17/8/025.

8. Lee YW, Kim M, Kim Y, Kang SW, Lee JH, Han SW. Synthesis and electrocatalytic activity of $\mathrm{Au}-\mathrm{Pd}$ alloy nanodendrites for ethanol oxidation.J Physic Chem C. 2010;114(17):7689-93. doi:10.1021/jp9119588.
9. Bagchi J, Bhattacharya SK. Electrocatalytic activity of binary palladium ruthenium anode catalyst on Ni-support for ethanol alkaline fuel cells. Transition Metal Chem. 2007;32(1):47-55. doi: 10.1007/s11243006-0127-9.

10. Wang Y, Zheng Y, Zhang L, Wang Q, Zhang D. Stability of nanosuspensions in drug delivery. J Control Release. 2013;172(3):1126-41. doi: 10.1016/j.jconrel.2013.08.006. [PubMed: 23954372].

11. Budroni G, Corma A. Gold-organic-inorganic high-surface-area materials as precursors of highly active catalysts. Angew Chem Int Ed Engl. 2006;45(20):3328-31. doi: 10.1002/anie.200600552. [PubMed: 16622895].

12. Anand P, Kunnumakkara AB, Newman RA, Aggarwal BB. Bioavailability of curcumin: Problems and promises. Mol Pharm. 2007;4(6):80718. doi: 10.1021/mp700113r. [PubMed:17999464].

13. Allegra A, Innao V, Russo S, Gerace D, Alonci A, Musolino C. Anticancer activity of curcumin and its analogues: Preclinical and clinical studies. Cancer Invest. 2017;35(1):1-22. doi: 10.1080/07357907.2016.1247166. [PubMed: 27996308].

14. Khaniki M, Azizian S, Alizadeh AM, Hemmati H, Emamipour N, Ali Mohagheghi M. The antiproliferative and anticancerogenic effects of nano-curcumin in rat colon cancer. Tehran Univ Med J. 2013;71(5).

15. Bengmark S. Curcumin, an atoxic antioxidant and natural NFkappaB, cyclooxygenase-2, lipooxygenase, and inducible nitric oxide synthase inhibitor: A shield against acute and chronic diseases.JPENJParenter Enteral Nutr. 2006;30(1):45-51. doi: 10.1177/014860710603000145. [PubMed: 16387899].

16. Alizadeh AM, Rohandel G, Roudbarmohammadi S, Roudbary M, Sohanaki H, Ghiasian SA, et al. Fumonisin B1 contamination of cereals and risk of esophageal cancer in a high risk area in northeastern Iran. Asian Pac J Cancer Prev. 2012;13(6):2625-8. doi: 10.7314/apjcp.2012.13.6.2625. [PubMed: 22938431]. 